\title{
PtAUREO1a and PtAUREO1b knockout mutants of the diatom Phaeodactylum tricornutum are blocked in photoacclimation to blue light ${ }^{\text {ir }}$
}

\author{
Marcus Mann ${ }^{\mathrm{a}, *}$, Manuel Serif ${ }^{\mathrm{b}}$, Torsten Jakob $^{\mathrm{a}}$, Peter G. Kroth ${ }^{\mathrm{b}}$, Christian Wilhelm ${ }^{\mathrm{a}}$ \\ a Institute of Biology, Department of Plant Physiology, University of Leipzig, D-04103 Leipzig, Germany \\ b Plant Ecophysiology, Fachbereich Biologie, Universität Konstanz, D-78457 Konstanz, Germany
}

\begin{abstract}
A B S T R A C T
Aureochromes are blue light receptors specifically found in photosynthetic Stramenopiles (algae). Four different Aureochromes have been identified in the marine diatom Phaeodactylum tricornutum (PtAUREO 1a, 1b, 1c, and 2). Since blue light is necessary for high light acclimation in diatoms, it has been hypothesized that Aureochromes might play an important role in the light acclimation capacity of diatoms. This hypothesis was supported by an RNAi knockdown line of PtAUREO1a, which showed a phenotype different from wild type cells when grown in either blue or red light. Here, we show for the first time the phenotype and the photoacclimation reaction of TALEN-mediated knockout mutants of PtAUREO1a and PtAUREO1b, clearly proving the necessity of Aureochromes for light acclimation under blue light. However, both mutants do also show specific differences in their respective phenotypes. Hence, PtAUREO1a and $1 \mathrm{~b}$ are not functionally redundant in photoacclimation to blue light, and their specific contribution needs to be clarified further.
\end{abstract}

\section{Introduction}

Diatoms are unique organisms being successful in extremely different aquatic environments. They are major primary producers in freshwater as well as marine environments, but also in the sediments in the nearshore and as ice algae growing in the ice edge in polar regions. Therefore, diatoms are important drivers of the global carbon and silicon biogeochemical cycles (Abrantes et al., 2016). In these different environments, the light climate can be very dynamic with respect to the oscillation in light intensity, but also in light quality and the duration of the light period (Depauw et al., 2012). For instance, in the polar regions the cells are exposed to permanent light in summer and long dark periods in winter, whereas in spring and autumn, when the water column is mixed, the ratio of blue light (BL) to red light (RL) can oscillate with high frequency. Considering that in photoautotrophic organisms the energy flux through the photosynthetic apparatus must be balanced with the metabolite flux in the dark reactions, which are controlled by temperature and nutrient availability, it is evident that diatoms need an extremely efficient light/ metabolite flux regulation with a fast responsiveness. Therefore, it is reasonable to suggest photoreceptors as key regulators for photoacclimation (Wilhelm et al., 2014).

In a previous study, Schellenberger Costa et al. (2013a) have shown that in the marine diatom Phaeodactylum tricornutum BL is essential for high light acclimation. Since light acclimation is a complex process including many well-orchestrated changes (especially in the metabolic network) (Fanesi et al., 2017), studying light-triggered transcription factors that may regulate several sets of genes in parallel, seems a good strategy.

The BL-regulated transcription factors Aureochromes have been discovered originally in the stramenopile alga Vaucheria frigida, in which they control photomorphogenesis, (Hisatomi et al., 2014; Takahashi et al., 2007). For diatoms, the structure and functional mechanisms of Aureochromes are well explored (Banerjee et al., 2016a; Heintz and Schlichting, 2016; Herman et al., 2013; Herman and Kottke, 2015), but little is known about their physiological function. So far, the only known physiological function of Aureochromes in $P$. tricornutum is the regulation of the onset of the cell cycle by PtAUREO1a, which is affecting the expression of the diatom-specific cyclin 2 (dsCYC2) (Huysman et al., 2013). It was further proposed that PtAUREO1a may be involved in the photoacclimation process and particularly in the regulation of the photoprotective potential (Schellenberger Costa et al., 2013a). Therefore, the generation of PtAUREO1a RNAi knockdown lines of $P$. tricornutum have been the first attempt to learn more on Aureochrome functions. Somewhat surprisingly, Schellenberger Costa et al. (2013b) could show that in these knockdown lines, photoacclimation was not impaired under BL conditions. However, in these mutants PtAUREO1a was reduced only to a level of slightly below $50 \%$

\footnotetext{
This article is part of a special issue entitled: Light driven reactions in model algae published at the Journal of Plant Physiology 217C.

* Corresponding author.

E-mail address: Marcus.Mann@uni-leipzig.de (M. Mann).
} 
of that of WT cells. Therefore, it is not clear if the observed effects were due to a reduced activity of PtAUREO1a alone, or resulted from compensatory activity of e.g. other regulatory factors, as has been described in other studies (Klier et al., 2008; Yang et al., 2013).

Hence, it was of high interest to study the photoacclimation of a TALEN-mediated PtAUREO1a knockout mutant (Serif et al., 2017) in response to cultivation under BL and RL. In addition, it was important to study the phenotype of a TALEN-mediated PtAUREO1b in comparison to a PtAUREO1a knockout mutant. The results of this comparison could provide valuable information about a putative functional redundancy of these Aureochromes in diatoms.

\section{Materials and methods}

\subsection{Cultivation}

P. tricornutum wild type (WT, UTEX646, University of Texas, USA), PtAUREO1a K8 and PtAUREO1b K5 knockout mutants were cultivated in modified artificial seawater medium f/2 (Guillard, 1975), at $20{ }^{\circ} \mathrm{C}$ and constantly bubbled with ambient air. Cultures were maintained in steady state by semi-continuous cultivation, with BL $(469 \pm 10 \mathrm{~nm})$ and RL (659 $\pm 11 \mathrm{~nm})$ LED panels as light source (CLF PlantClimatics, Germany), under a 14/10 h light/dark regime. The incident irradiance was adjusted to yield the same amount of photosynthetically absorbed radiation $\left(\mathrm{Q}_{\text {phar }}\right.$; calculated according to Gilbert et al. (2000)) under RL and BL conditions, respectively. Accordingly, an incident irradiance of $100 \mu \mathrm{mol}$ photons $\mathrm{m}^{-2} \mathrm{~s}^{-1} \mathrm{RL}$ and $60 \mu \mathrm{mol}$ photons $\mathrm{m}^{-2} \mathrm{~s}^{-1} \mathrm{BL}$ yielded a $Q_{p h a r}$ of $30 \mu \mathrm{mol}$ photons $\mathrm{m}^{-2} \mathrm{~s}^{-1}$, respectively. In this way, any overlaying effects of light quality and light quantity are avoided. Cultures were acclimated to steady state conditions for ten days before starting the experimental procedures.

\subsection{Aureochrome knockout mutants}

A TALEN-mediated PtAUREO1a knockout mutant has been generated prior to this study (Serif et al., 2017). TALEN plasmids against PtAureo1b were designed and constructed according to the same guidelines. The TALEN pair chosen has no predicted potential off-targets, is predicted to possess a high cleavage activity and cuts at position 422 downstream of the start codon of PtAureo1b and targets the sequence $5^{\prime}$-TCAGCAAACACAGAATGCTT- $3^{\prime}$ on the sense strand and $5^{\prime}$ TTGTACCATCGCGGCACCCG- $3^{\prime}$ on the antisense strand. Nuclear transformation of $P$. tricornutum was performed using a Bio-Rad Biolistic PDS-1000/He Particle Delivery System (Bio-Rad, Hercules, CA, USA) fitted with $900 / 1100 / 1350$ psi rupture disks as described previously (Kroth, 2007; Zaslavskaia et al., 2000). $10^{8}$ cells per plate were bombarded with $1.25 \mu \mathrm{g}$ of each plasmid. For selective cultivation of $P$. tricornutum transformants, $75 \mathrm{mg} \mathrm{mL}^{-1}$ Zeocin (Invitrogen, Carlsbad, CA, USA) and $150 \mathrm{mg} \mathrm{mL}^{-1}$ Nourseothricin (ClonNat, Werner Bioagents, Jena, Germany) were added to solid f/2 media (Zaslavskaia et al., 2000). Colonies obtained after transformation were screened for targeted mutagenesis events by PCR (Supplementary Fig. S1) (primers used: 5'-TTGAACGAAATCTTTGCCGAGTA-3' and 5'-TGCGTTTGGCGTGTTCAC-3') and Western Blot (Fig. 1) using custom antiserum against

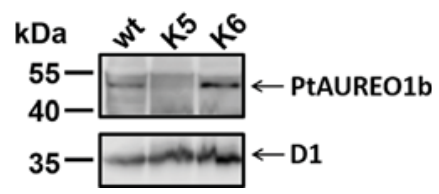

Fig. 1. Western Blot of $P$. tricornutum WT and two transformants, using a PtAUREO1bspecific antiserum. The PtAUREO1b K5 knockout mutant was used for this study, with no residual protein detectable. No reduction of PtAUREO1b was detected in strain K6. The expected molecular weight of PtAUREO1b is $46 \mathrm{kDa}$. A D1-specific antibody was used as a loading control.
PtAUREO1b (Agrisera AB, Vännas, Sweden) with antiserum against D1protein (AS05-084, Agrisera AB) as a loading control as described for PtAUREO1a in Serif et al. (2017).

\subsection{Cellular parameters}

The concentration of Chlorophyll a (Chl $a$ ) was determined by collecting cells on cellulose filters (MN 85/70, Macherey-Nagel, Germany) and extraction in $90 \%$ acetone by using a tissue grinder (Precellys24, Bertin technologies, France). Chl $a$ concentration was determined spectrophotometrically at wavelength 664 and $630 \mathrm{~nm}$ according to Jeffrey and Humphrey (1975) with $90 \%$ acetone as blank $\left(\mathrm{E}_{750 \mathrm{~nm}}=0\right)$.

The Chl $a$ absorption coefficient (a* phy) was calculated from the in vivo absorption spectra and $\mathrm{Chl} a$ concentration of acclimated cells, according to Gilbert et al. (2000).

Dry weight was determined by means of weighing by difference. Accordingly, cells were collected on a pre-weighed polycarbonate filter (pore size $0.2 \mu \mathrm{m}$, GVS life sciences, USA) and washed with distilled water. The filters were weighed again after $24 \mathrm{~h}$ of freeze drying (FreeZone 2.5, LABCONCO, USA).

Cell counts were determined using a Bürker type Haemocytometer (Marienfeld, Germany).

\subsection{Photosynthetic parameters}

Photosynthetic parameters were measured using a specially equipped Light pipette (Illuminova, Sweden). In a custom sample cuvette, oxygen based photosynthesis rates were measured with a Clark-type electrode (MI-730, Microelectrodes Inc., USA) and, simultaneously, non-photochemical quenching (NPQ) was determined via Pulse Amplitude Modulated (PAM) fluorometry (PAM 101, WALZ $\mathrm{GmbH}$, Germany). For a more detailed description of this device and calculations, see (Blache et al., 2011). Photosynthetic parameters of BL and RL acclimated cells were measured under illumination with white light (halogen 64642HLX, Osram, Germany) according to Schellenberger Costa et al. (2013a).

\section{Results}

A knockout mutant of isoform PtAUREO1b was generated using TALEN (PtAUREO1b K5). It was confirmed via Western Blot, using an isoform-specific antiserum, that no PtAUREO1b protein was detectable in this cell line (Fig. 1). To investigate a putative functional redundancy of the Aureochromes, we compared this strain to a previously generated PtAUREO1a knockout mutant (PtAUREO1a K8) (Serif et al., 2017). For a general characterisation of the photoacclimation status of WT and mutant cells under steady state RL and BL conditions, basic cell parameters were determined, and photosynthesis-irradiance curves (PIcurves) and the corresponding values of non-photochemical quenching of Chl fluorescence were recorded.

\subsection{Steady state $R L$}

The optical properties of phytoplankton cells basically depend on their cellular Chl $a$ content. From Table 1 it is evident that under RL conditions WT and mutants possessed comparable amounts of Chl $a$ per cell. The slightly lower $\mathrm{Chl} a$ content in cells of PtAUREO1a K8 resulted in a correspondingly increased $\mathrm{Chl} a$ absorption coefficient (a* phy) compared to WT cells and PtAUREO1b K5. The PI-curves and the NPQ values did reveal minor differences between WT and mutant cells of $P$. tricornutum. The highest maximum photosynthesis rates $\left(\mathrm{P}_{\max }\right)$ were measured in the WT ( $284 \mu \mathrm{mol} \mathrm{O}_{2} \mathrm{mg} \mathrm{Chl} a^{-1} \mathrm{~h}^{-1}$ ), whereas $\mathrm{P}_{\max }$ in PtAUREO1a K8 and PtAUREO1b K5 were slightly lower (23\% and 14\%, respectively; Fig. 2a). In contrast, the highest values for maximum non-photochemicalquenching $\left(\mathrm{NPQ}_{\max }\right)$ of $\sim 0.55$ were observed in both mutants, whereas the WT showed a slightly lower $\mathrm{NPQ}_{\max }$ of 0.43 (Fig. 3a). 
Table 1

Cellular Parameters of P. tricornutum WT, PtAUREO1a K8 and PtAUREO1b K5 knockout mutants acclimated to steady state red- and blue light. Mean values are shown with standard deviation $(n=4)$. Significant differences within each parameter were tested with a one-way analysis of variance (ANOVA) followed by a Holm-Sidak all pairwise multi comparison test. Values that are not significantly different are marked with the same letter $(\mathrm{P}<0.05)$.

\begin{tabular}{|c|c|c|c|c|c|c|}
\hline & \multicolumn{3}{|l|}{ RL } & \multicolumn{3}{|l|}{ BL } \\
\hline & WT & PtAUREO1a K8 & PtAUREO1b K5 & WT & PtAUREO1a K8 & PtAUREO1b K5 \\
\hline Chl $a$ per cell [pg Chl $a$ cell $\left.{ }^{-1}\right]$ & $0.47 \pm 0.01^{\mathrm{ac}}$ & $0.42 \pm 0.03^{\mathrm{b}}$ & $0.48 \pm 0.02^{\mathrm{cd}}$ & $0.41 \pm 0.02^{\mathrm{b}}$ & $0.47 \pm 0.03^{\mathrm{ad}}$ & $0.63 \pm 0.01^{\mathrm{e}}$ \\
\hline $\mathrm{a}^{*}$ phy $\left[\mathrm{m}^{2} \mathrm{~g} \mathrm{Chl} a^{-1}\right]$ & $11.3 \pm 0.2^{\mathrm{a}}$ & $12.2 \pm 0.2^{\mathrm{d}}$ & $10.3 \pm 0.2^{\mathrm{b}}$ & $11.7 \pm 0.1^{\mathrm{c}}$ & $11.1 \pm 0.1^{\mathrm{a}}$ & $10.0 \pm 0.2^{\mathrm{b}}$ \\
\hline dry weight $\left[\mathrm{pg}\right.$ cell $\left.^{-1}\right]$ & $32.5 \pm 2.0^{\mathrm{cegh}}$ & $35.7 \pm 3.2^{\mathrm{bdg}}$ & $34.1 \pm 3.6^{\mathrm{acd}}$ & $24.8 \pm 2.0^{\mathrm{f}}$ & $27.6 \pm 3.0^{\mathrm{fh}}$ & $34.5 \pm 1.7^{\text {abe }}$ \\
\hline
\end{tabular}
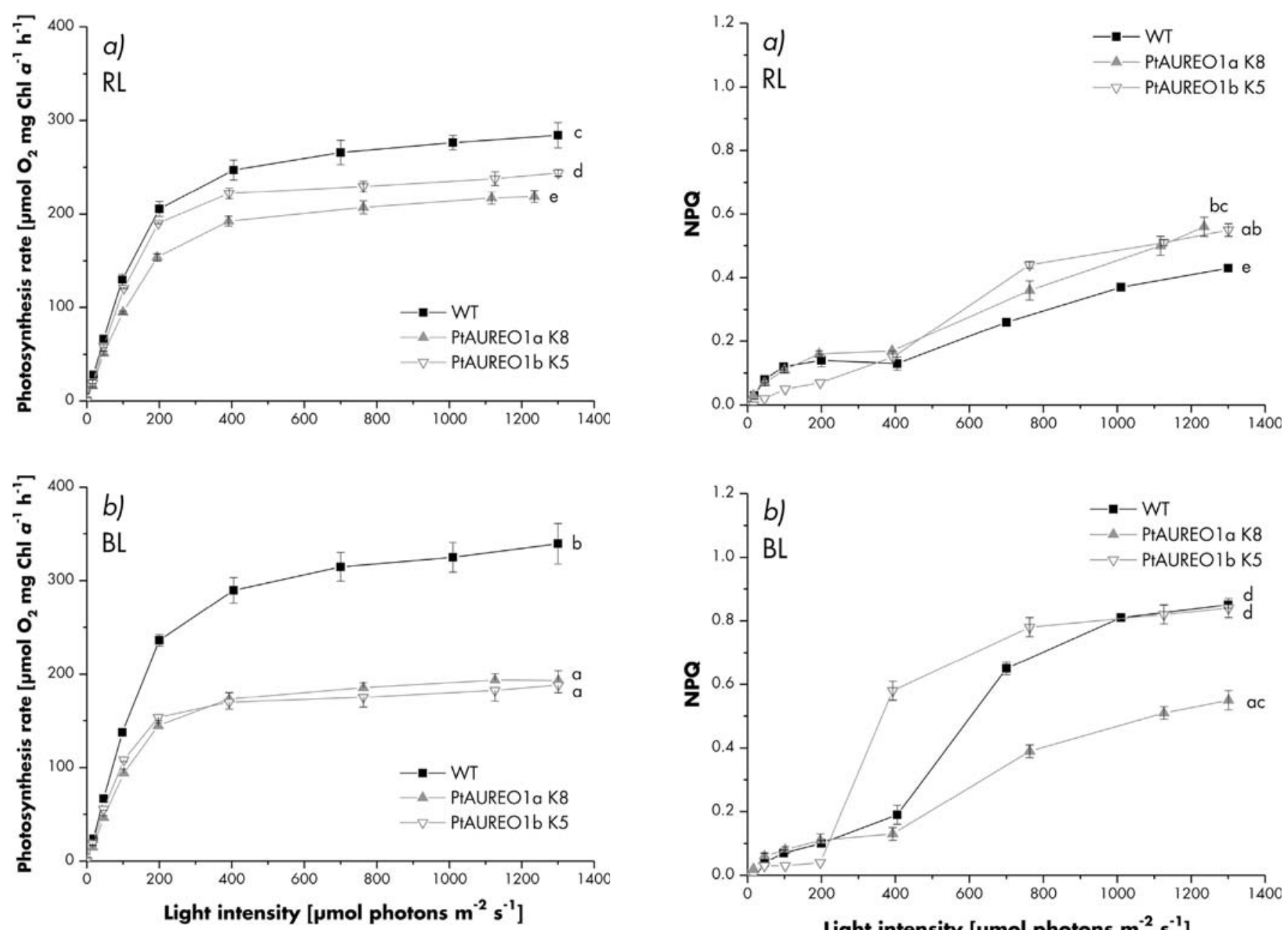

Fig. 2. Photosynthesis-irradiance curves (PI-curves) of P. tricornutum WT, PtAUREO1a K8 and PtAUREO1b K5 knockout mutants acclimated to steady state red- (a) and blue light (b). The corresponding light intensities, display the incident light applied to the measuring cuvette. Mean values are shown with standard deviation $(n=4)$. Significant differences between maximum photosynthesis rates were tested with a one-way analysis of variance (ANOVA) followed by a Holm-Sidak all pairwise multi comparison test. Values that are not significantly different are marked with the same letter $(\mathrm{P}<0.05)$.

\subsection{Steady state $B L$}

In contrast to RL, the cultivation under BL conditions induced much greater differences in the photoacclimation status of the WT compared to PtAUREO1a K8 and PtAUREO1b K5. In both mutants, an increase of the cellular Chl $a$ content and a decrease of a* phy was observed in comparison to WT cells (Table 1).

The most striking differences between WT and mutant strains were observed in PI-Curves and NPQ values under BL with $44 \%$ lowered $\mathrm{P}_{\max }$ values for both mutants compared to the WT cells (Fig. 2b). While PtAUREO1a K8 showed a significantly lower $\mathrm{NPQ}_{\max }$ of 0.58 compared to

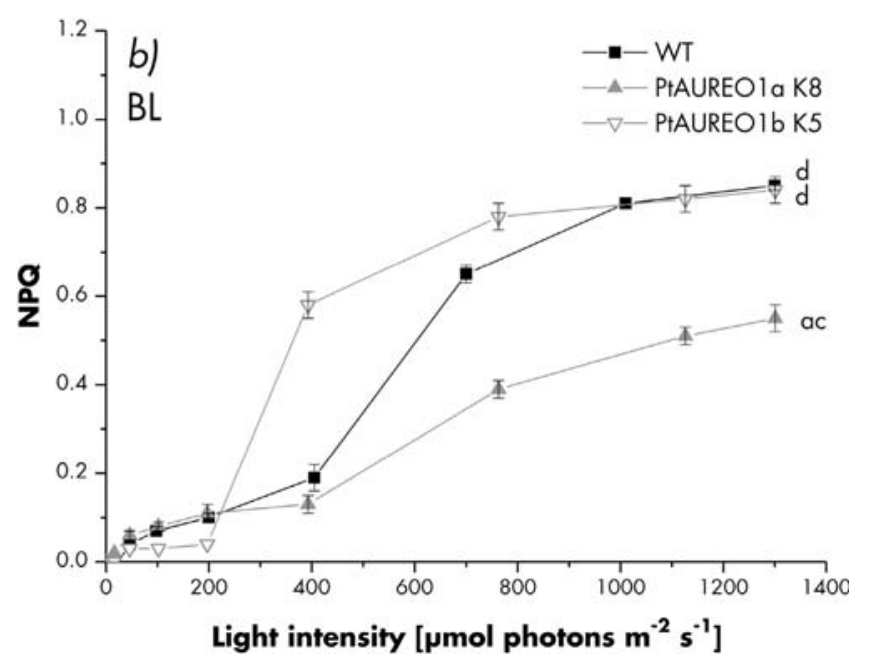

Fig. 3. Non-photochemical quenching (NPQ) induction of $P$. tricornutum WT, PtAUREO1a K8 and PtAUREO1b K5 knockout mutants acclimated to steady state red- (a) and blue light (b). The corresponding light intensities, display the incident light applied to the measuring cuvette. Mean values are shown with standard deviation $(n=4)$. Significant differences between $\mathrm{NPQ}_{\max }$ values were tested with a one-way analysis of variance (ANOVA) followed by a Holm-Sidak all pairwise multi comparison test. Values that are not significantly different are marked with the same letter $(\mathrm{P}<0.001)$.

$\sim 0.85$ in WT and PtAUREO1b K5 (Fig. 3b), a different pattern of irradiance-dependence of NPQ induction was observed in PtAUREO1b K5. Here, the induction of NPQ started already at an irradiance of $>200$ $\mu \mathrm{mol}$ photons $\mathrm{m}^{-2} \mathrm{~s}^{-1}$, whereas NPQ induction in WT and PtAUREO1a $\mathrm{K} 8$ was observed at an irradiance of $>400 \mu \mathrm{mol}$ photons $\mathrm{m}^{-2} \mathrm{~s}^{-1}$.

\section{3. $B L v s . R L$}

Whereas the cellular Chl $a$ content in WT cells decreased in BL compared to the RL, an increase was observed in both Aureochrome 
knockout mutants, with a higher incidence of this effect in PtAUREO1b K5. As a consequence, a* phy is slightly increased in the WT cells, and decreased in PtAUREO1a K8, while it is not changed in PtAUREO1b K5 (Table 1). Again, striking differences were observed in the PI-curves and the NPQ values. In the WT cells, $\mathrm{P}_{\max }$ under BL increased significantly by $19 \%$ in comparison to WT cells under RL conditions (Fig. 2b). In contrast, $\mathrm{P}_{\max }$ of PtAUREO1a K8 and PtAUREO1b K5 decreased by 12 and $23 \%$, respectively, in the comparison of BL $v s$. RL conditions (Fig. 2). The $\mathrm{NPQ}_{\max }$ value of the WT cells increased by a factor of 2 from 0.43 under BL to 0.85 under RL. In contrast, there was no change of $\mathrm{NPQ}_{\max }$ of PtAUREO1a K8, but a drastic increase of $\mathrm{NPQ}_{\max }$ by a factor of 1.5 in PtAUREO1b K5 (Fig. 3).

\section{Discussion}

\subsection{PtAUREO1 $a$ and $1 b$ are indispensable for photoacclimation under $B L$}

The study of Schellenberger Costa et al. (2013a) showed that BL is essential for HL acclimation in WT cells, as evidenced by higher values of $\mathrm{P}_{\max }$ and $\mathrm{NPQ}_{\max }$ under BL in regard to RL. This is confirmed by the results of the present study. In addition, it was shown that PtAUREO1a and $1 \mathrm{~b}$ knockout mutants, respectively, possess a significantly lower potential of photosynthesis ( $\mathrm{P}_{\max }$ values) under BL steady state conditions compared to the WT cells. Furthermore, PtAUREO1a K8 showed a significantly lower potential of light protection (NPQ $\mathrm{Nax}_{\max }$ values) in comparison to the WT. A different photoacclimation status in comparison of the Aureochrome knockout mutants and the WT is also indicated by the significantly higher Chl content per cell and lower $a^{*}$ phy in PtAUREO1a K8 and PtAUREO1b K5 vs. WT cells. Both parameters typically change in response to acclimation to different irradiation intensities (Blache et al., 2011; Wilhelm et al., 2014). Taken together, it can be concluded that under BL conditions the WT cells were in a high light-acclimated state, whereas the Aureochrome-deficient mutants were arrested in a low light-acclimated state. In contrast, under RL there were only minor differences in photosynthetic parameters between WT cells and Aureochrome knockout mutants. Consequently, this clearly indicates an absence of the ability to photoacclimate to $\mathrm{BL}$ conditions in PtAUREO1a K8 and PtAUREO1b K5. Thus, PtAUREO1a and $1 \mathrm{~b}$ are obviously indispensable for the perception of BL and probably also for the signal transduction required for photoacclimation.

\subsection{Functional redundancy of aureochromes}

Based on current knowledge, it is still unclear whether the four Aureochromes in P. tricornutum might be functionally redundant. Based on the results in this study, it is evident that PtAUREO1a K8 and PtAUREO1b K5 showed specific differences in NPQ under BL condition. This could indicate that PtAUREO1a and 1b might not be functionally redundant. Whereas $\mathrm{NPQ}_{\max }$ values were similar in PtAUREO1a K8 and PtAUREO1b K5 under RL conditions, a significantly higher NPQ ${ }_{\max }$ was observed in PtAUREO1b K5 under BL (similar to WT) in comparison to PtAUREO1a K8. In addition, the kinetic of NPQ induction was significantly different under BL in PtAUREO1b K5 compared to PtAUREO1a K8 and WT cells. NPQ is a protective mechanism against excessive light, thereby preventing e.g. the formation of reactive oxygen species (ROS). In diatoms, a strong connection between NPQ and LHCX proteins is well known (Goss and Lepetit, 2015). Whereas LHCX1 affects the overall capacity of NPQ, it is speculated that LHCX2 and LHCX3 have an influence on the efficiency of photoprotection (Bailleul et al., 2010; Lepetit et al., 2013). For the WT, Schellenberger Costa et al. (2013a) were able to show that a higher $\mathrm{NPQ}_{\max }$ under BL compared to RL was accompanied by an increase of the LHCX1 protein. In the same study, it was shown that the promoter region of the LHCX1 gene possesses two potential binding sites for PtAUREO1a. Thus, together with the results of the present study, it can be concluded that PtAUREO1a, but not PtAUREO1b, is involved in the regulation of the maximum capacity of photoprotection by NPQ. In addition, there is further support for the assumption that PtAUREO1a is involved in the regulation of the expression level of the LHCX1 protein.

Taken together, we propose that PtAUREO1a and $1 \mathrm{~b}$ are both involved in photoacclimation under BL, however, they may regulate different aspects of photoacclimation, particularly with respect to NPQ formation. Future studies of potential changes of transcriptomes under different light quality conditions might help to elucidate the specific role of PtAUREO1a and $1 \mathrm{~b}$ in the regulation of gene expression of proteins related to e.g. photoprotection.

\subsection{Role of Aureochromes in photoacclimation under $R L$}

Besides striking differences in the photoacclimation of PtAUREO1a and $1 \mathrm{~b}$ knockout mutants under BL compared to WT cells, significant differences were discovered also with respect to acclimation to RL. This is evidenced by a slightly higher $\mathrm{NPQ}_{\max }$ and a lower $\mathrm{P}_{\max }$ in both mutants compared to the WT, which is in line with other studies that report BL-independent effects regarding Aureochromes. Recently, Banerjee et al. (2016b) showed that PtAureo1a is expressed in a light independent circadian rhythm and that the bZIP domain of PtAUREO1a is capable of binding DNA also in the dark. Additionally, it was discovered that the PtAUREO1a LOV domain is able to form dimers in the dark (Banerjee et al., 2016a; Heintz and Schlichting, 2016). The potential functional difference between this dark-state dimer and the previously described BL-induced dimerization of Aureochrome LOV domains (Herman et al., 2013; Herman and Kottke, 2015) remains unclear. Along with our data, it seems reasonable to suggest BL-independent functions of Aureochromes, but further investigation is necessary to elucidate this assumption.

\section{Acknowledgements}

This work was funded by the Deutsche Forschungsgemeinschaft (DFG), as part of the DFG research group FOR 1261 (Grants Kr1661/8-1 and 8-2 to PGK, and grants Wi764/8-2 and 8.2 to CW). PGK is furthermore thankful for support by the Graduate School Biological Sciences (GBS) of the University of Konstanz.

\section{References}

Abrantes, F., Cermeno, P., Lopes, C., Romero, O., Matos, L., Van Iperen, J., Rufino, M., Magalhães, V., 2016. Diatoms Si uptake capacity drives carbon export in coastal upwelling systems. Biogeosciences 13, 4099-4109. http://dx.doi.org/10.5194/bg13-4099-2016.

Bailleul, B., Rogato, A., de Martino, A., Coesel, S., Cardol, P., Bowler, C., Falciatore, A., Finazzi, G., 2010. An atypical member of the light-harvesting complex stress-related protein family modulates diatom responses to light. Proc. Natl. Acad. Sci. 107, 18214-18219. http://dx.doi.org/10.1073/pnas.1007703107.

Banerjee, A., Herman, E., Kottke, T., Essen, L.O., 2016a. Structure of a native-like aureochrome 1a LOV domain dimer from phaeodactylum tricornutum. Structure 24 , 171-178. http://dx.doi.org/10.1016/j.str.2015.10.022.

Banerjee, A., Herman, E., Serif, M., Maestre-Reyna, M., Hepp, S., Pokorny, R., Kroth, P.G., Essen, L.O., Kottke, T., 2016b. Allosteric communication between DNA-binding and light-responsive domains of diatom class i aureochromes. Nucleic Acids Res. 44, 5957-5970. http://dx.doi.org/10.1093/nar/gkw420.

Blache, U., Jakob, T., Su, W., Wilhelm, C., 2011. The impact of cell-specific absorption properties on the correlation of electron transport rates measured by chlorophyll fluorescence and photosynthetic oxygen production in planktonic algae. Plant Physiol. Biochem. 49, 801-808. http://dx.doi.org/10.1016/j.plaphy.2011.04.010.

Depauw, F.A., Rogato, A., D'Alcalá, M.R., Falciatore, A., 2012. Exploring the molecular basis of responses to light in marine diatoms. J. Exp. Bot. 63, 1575-1591. http://dx. doi.org/10.1093/jxb/ers005.

Fanesi, A., Wagner, H., Wilhelm, C., 2017. Phytoplankton growth rate modelling: can spectroscopic cell chemotyping be superior to physiological predictors? Proc. Biol. Sci. 284. http://dx.doi.org/10.1098/rspb.2016.1956. 
Gilbert, M., Domin, A., Becker, A., Wilhelm, C., 2000. Estimation of primary productivity by chlorophyll a in vivo fluorescence in freshwater phytoplankton. Photosynthetica 38, 111-126. http://dx.doi.org/10.1023/A:1026708327185.

Goss, R., Lepetit, B., 2015. Biodiversity of NPQ. J. Plant Physiol. 172, 13-32. http://dx. doi.org/10.1016/j.jplph.2014.03.004.

Guillard, R.R.L., 1975. Culture of phytoplankton for feeding marine invertebrates. In: Smith, W.L., Chanley, M.H. (Eds.), Culture of Marine Invertebrate Animals. Springer, US, Boston MA, pp. 29-60. http://dx.doi.org/10.1007/978-1-4615-8714-93.

Heintz, U., Schlichting, I., 2016. Blue light-induced LOV domain dimerization enhances the affinity of aureochrome 1a for its target DNA sequence. Elife 5, e11860. http:// dx.doi.org/10.7554/eLife.11860.

Herman, E., Kottke, T., 2015. Allosterically regulated unfolding of the A' $\alpha$ helix exposes the dimerization site of the blue-light-sensing aureochrome-LOV domain. Biochemistry 54, 1484-1492. http://dx.doi.org/10.1021/bi501509z.

Herman, E., Sachse, M., Kroth, P.G., Kottke, T., 2013. Blue-light-induced unfolding of the $\mathrm{J} \alpha$ helix allows for the dimerization of aureochrome-LOV from the diatom phaeodactylum tricornutum. Biochemistry 52, 3094-3101. http://dx.doi.org/10.1021/ bi400197u.

Hisatomi, O., Nakatani, Y., Takeuchi, K., Takahashi, F., Kataoka, H., 2014. Blue lightinduced dimerization of monomeric Aureochrome-1 enhances its affinity for the target sequence. J. Biol. Chem. 289, 17379-17391. http://dx.doi.org/10.1074/jbc. M114.554618.

Huysman, M.J.J., Fortunato, A.E., Matthijs, M., Costa, B.S., Vanderhaeghen, R., Van den Daele, H., Sachse, M., Inzé, D., Bowler, C., Kroth, P.G., Wilhelm, C., Falciatore, A., Vyverman, W., De Veylder, L., 2013. AUREOCHROME1a-mediated induction of the diatom-specific cyclin dsCYC2 controls the onset of cell division in diatoms (Phaeodactylum tricornutum). Plant Cell 25, 1-15. http://dx.doi.org/10.1105/tpc 112.106377.

Jeffrey, S.W., Humphrey, G.F., 1975. New spectrophotometric equations for determining chlorophylls a1, b1, c1 and c2 in higher plants, algae and natural phytoplankton. Biochem. Physiol. Pflanz. 167, 191-194.

Klier, M., Anastasov, N., Hermann, A., Meindl, T., Angermeier, D., Raffeld, M., Fend, F., Quintanilla-Martinez, L., 2008. Specific lentiviral shRNA-mediated knockdown of cyclin D1 in mantle cell lymphoma has minimal effects on cell survival and reveals a regulatory circuit with cyclin D2. Leukemia 22, 2097-2105. http://dx.doi.org/10. 1038/leu.2008.213.

Kroth, P.G., 2007. Genetic transformation: a tool to study protein targeting in diatoms. In: van der Giezen, M. (Ed.), Methods in Molecular Biology. Humana Press, Totowa NJ, pp. 257-267. http://dx.doi.org/10.1007/978-1-59745-466-717.

Lepetit, B., Sturm, S., Rogato, A., Gruber, A., Sachse, M., Falciatore, A., Kroth, P.G., Lavaud, J., 2013. High light acclimation in the secondary plastids containing diatom Phaeodactylum tricornutum is triggered by the redox state of the plastoquinone pool. Plant Physiol. 161, 853-865. http://dx.doi.org/10.1104/pp.112.207811.

Schellenberger Costa, B., Jungandreas, A., Jakob, T., Weisheit, W., Mittag, M., Wilhelm, C., 2013a. Blue light is essential for high light acclimation and photoprotection in the diatom Phaeodactylum tricornutum. J. Exp. Bot. 64, 483-493. http://dx.doi.org/10. 1093/jxb/ers340.

Schellenberger Costa, B., Sachse, M., Jungandreas, A., Bartulos, C.R., Gruber, A., Jakob, T., Kroth, P.G., Wilhelm, C., 2013b. Aureochrome 1a is involved in the photoacclimation of the diatom phaeodactylum tricornutum. PLoS One 8, e74451. http:// dx.doi.org/10.1371/journal.pone.0074451.

Serif, M., Lepetit, B., Weißert, K., Kroth, P.G., Rio Bartulos, C., 2017. A fast and reliable strategy to generate TALEN-mediated gene knockouts in the diatom Phaeodactylum tricornutum. Algal Res. 23, 186-195. http://dx.doi.org/10.1016/j.algal.2017.02. 005 .

Takahashi, F., Yamagata, D., Ishikawa, M., Fukamatsu, Y., Ogura, Y., Kasahara, M., Kiyosue, T., Kikuyama, M., Wada, M., Kataoka, H., 2007. AUREOCHROME, a photoreceptor required for photomorphogenesis in stramenopiles. Proc. Natl. Acad. Sci. U. S. A. 104, 19625-19630. http://dx.doi.org/10.1073/pnas.0707692104.

Wilhelm, C., Jungandreas, A., Jakob, T., Goss, R., 2014. Light acclimation in diatoms: from phenomenology to mechanisms. Mar. Genomics 16, 5-15. http://dx.doi.org/10. 1016/j.margen.2013.12.003.

Yang, X., Cao, P., Südhof, T.C., 2013. Deconstructing complexin function in activating and clamping $\mathrm{Ca} 2+$-triggered exocytosis by comparing knockout and knockdown phenotypes. Proc. Natl. Acad. Sci. U. S. A. 110, 20777-20782. http://dx.doi.org/10. 1073/pnas.1321367110.

Zaslavskaia, L.A., Casey Lippmeier, J., Kroth, P.G., Grossman, A.R., Apt, K.E., 2000. Transformation of the diatom Phaeodactylum tricornutum (Bacillariophyceae) with a variety of selectable marker and reporter genes. J. Phycol. 36, 379-386. http://dx. doi.org/10.1046/j.1529-8817.2000.99164.x. 